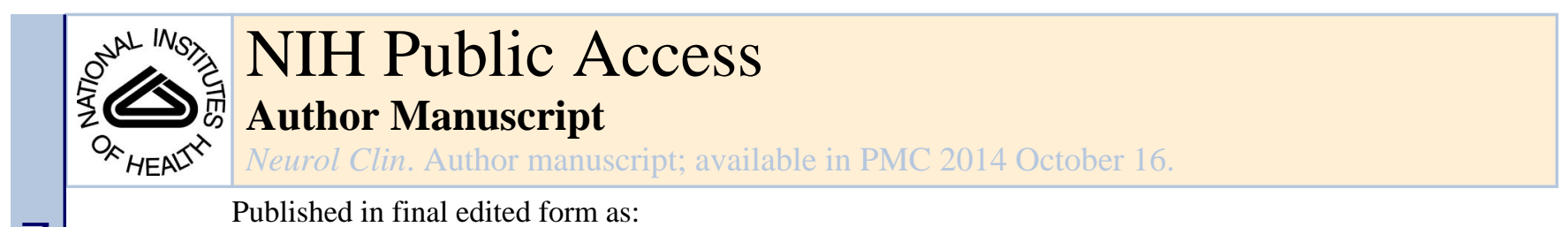

Published in final edited form as:

Neurol Clin. 2013 May ; 31(2): 477-489. doi:10.1016/j.ncl.2013.02.002.

\title{
Nutritional Neuropathies
}

Nancy Hammond, MD, Yunxia Wang, MD, Mazen Dimachkie, MD, and Richard Barohn, MD University of Kansas Medical Center, Kansas City, KS

Yunxia Wang: ywang@kumc.edu

\section{Keywords}

Neuropathy; Thiamine; Vitamin B12; Copper; Vitamin B6; Bariatric Surgery

\section{Introduction}

Malnutrition can affect all areas of the nervous system. Risk factors for malnutrition include alcohol abuse, eating disorders, older age, pregnancy, homelessness, and lower economic status. Any medical condition that affects the GI tract can also impair absorption of essential vitamins. Nutritional deficiencies have been described in patients with inflammatory bowel disease, fat malabsorption, chronic liver disease, pancreatic disease, gastritis, and small bowel resections. Patients receiving total parental nutrition (TPN) are also at risk for vitamin deficiency and TPN formulations should be carefully formulated to include supplemental vitamins and trace minerals. Neurological complications following gastric bypass surgery are increasingly recognized. Nutritional neuropathies manifest either acutely, subacutely, or chronically. They can be either demyelinating or axonal.

A unique class of peripheral neuropathy with coexistent myelopathy, also called myeloneuropathy, can also been seen with nutritional neuropathies. Myeloneuropathy has been described with deficiencies of vitamin B12 and copper.

Patients with myeloneuropathy will present with both upper motor neuron and lower motor neuron signs. Peripheral neuropathy may mask the symptoms and signs of the myelopathy presenting a diagnostic challenge. Hyper reflexia may be difficult to assess in the presence of severe peripheral neuropathy and ankle jerks may be absent. Muscle weakness may impair the toe extensors, so Babinski sign may not be present. Besides spinal cord/cauda equina arteriovenous malformation, the clinician should suspect myeloneuropathy when the predominant complaint is gait impairment or bowel or bladder dysfunction in the setting of a peripheral neuropathy.

(C) 2013 Elsevier Inc. All rights reserved.

Publisher's Disclaimer: This is a PDF file of an unedited manuscript that has been accepted for publication. As a service to our customers we are providing this early version of the manuscript. The manuscript will undergo copyediting, typesetting, and review of the resulting proof before it is published in its final citable form. Please note that during the production process errors may be discovered which could affect the content, and all legal disclaimers that apply to the journal pertain. 


\section{Thiamine Deficiency}

\section{Pathogenesis}

Thiamine (vitamin B1) is a water-soluble vitamin present in most animal and plant tissues. Neuropathy due to thiamine deficiency, known as beriberi, was the first clinically described deficiency syndrome in humans. Beriberi may manifest with heart failure (wet beriberi) or without heart failure (dry beriberi). Thiamine deficiency is also responsible for Wernicke's encephalopathy and Korsakoff's syndrome. Thiamine is absorbed in the small intestine by both passive diffusion and active transport and rapidly converted to thiamine diphosphate (TDP). TDP serves as an essential co-factor in cellular respiration, ATP production, synthesis of glutamate and $\gamma$-aminobutyric acid[1] and myelin sheath maintenance. Only about 20 days of thiamine are stored in the body, and thiamine deficiency can start to manifest in as little as three weeks. The recommended daily allowance (RDA) for thiamine ranges from $1.0 \mathrm{mg}$ per day for young healthy adults to $1.5 \mathrm{mg}$ per day for breastfeeding women[2]. Athletes and patients with higher metabolic needs as seen during pregnancy, systemic infections, and certain cancers need a higher daily intake of thiamine. Thiamine deficiency is rare in industrialized countries and is most commonly seen in the setting of chronic alcohol abuse, recurrent vomiting, AIDS, long-term total parenteral nutrition, eating disorders and weight reduction surgery.

\section{Clinical Features}

Symptoms usually develop gradually over weeks to months, but sometimes they may manifest rapidly over a few days mimicking Gullain Barré Syndrome[3, 4]. Fatigue, irritability, and muscle cramps may appear within days to weeks of nutritional deficiency[5]. Clinical features of thiamine deficiency begin with distal sensory loss, burning pain, paraesthesias or muscle weakness in the toes and feet[6]. There is often associated aching and cramping in the lower legs. Left untreated, the neuropathy will cause ascending weakness in the legs and eventually evolve to a sensorimotor neuropathy in the hands. Beriberi may include involvement of the recurrent laryngeal nerve, producing hoarseness and cranial nerve involvement manifesting as tongue and facial weakness [7]. Oculomotor muscle weakness and nystagmus have been attributed to beriberi, but these manifestations are more likely due to coexistent Wernicke's disease. Approximately 25\% of patients with thiamine deficient polyneuropathy may also have Wernicke's encephalopathy which manifests as ophthalmoplegia, ataxia, nystagmus and encephalopathy[6].

\section{Diagnosis}

Blood and urine assays for thiamine are not reliable for diagnosis of deficiency. Measurement of thiamine pyrophosphate by high-performance liquid chromatography[8] or erythrocyte transketolase activation may be preferred for assessment of thiamine status[9]. However, the precise sensitivity and specificity for those assays has not been established. Testing must be performed before thiamine supplementation is given. Electrodiagnostic testing shows an axonal sensorimotor polyneuropathy worse in the lower extremities and nerve biopsies demonstrate axonal degeneration [6]. 


\section{Management}

When a diagnosis of thiamine deficiency is made or suspected, thiamine replacement should be provided until proper nutrition is restored. Thiamine is usually given intravenously or intramuscularly at an initial dose of $100 \mathrm{mg}$ followed by $100 \mathrm{mg}$ per day. Cardiac manifestations may improve within hours to days while neurological improvement may take 3-6 months with motor manifestations responding better than sensory symptoms[10]. Some improvement is expected in most patients, but this typically occurs slowly, and in patients with severe neuropathy, there may be permanent deficits[11].

\section{Vitamin B12}

\section{Pathogenesis}

Vitamin B12 (cobalamin) is present in animal and dairy products and is synthesized by specific microorganisms. Humans depend on nutritional intake for their vitamin B12 supply. Vitamin B12 deficiency has been observed in 5\% to $20 \%$ of older adults and up to $40 \%$ of older adults have low serum vitamin B12 levels[12]. The RDA for vitamin B12 is $2.4 \mathrm{mcg}$ daily[2].

Vitamin B12 is an integral component of two biochemical reactions in human. The first is the formation of methionine by methylation of homocysteine. A byproduct of this reaction is the formation of tetrahydrofolate, an important precursor of purine and pyrimidine synthesis. The second important reaction is the conversion of L-methylmalonyl coenzyme A into succinyl coenzyme A which is essential for formation of the myelin sheath.

Vitamin B12 is liberated from food by stomach acid and pepsin. Liberated B12 then binds to $R$ proteins secreted in the saliva and gastric secretions. Cobalamin is released from the $R$ protein in the small intestine and binds to intrinsic factor. The vitamin B12-intrisic factor complex is then absorbed in the terminal ileum.

Cases of vitamin B12 deficiency can be due to malabsorption, pernicious anemia, gastrointestinal surgeries and weight reduction surgery. As vitamin B12 is only found in animal products strict vegan diets lack vitamin B12 and must be supplemented. Certain medications may contribute to vitamin B12 deficiency namely proton pump inhibitors[13] and metformin[14]. An underappreciated cause of $\mathrm{Cbl}$ deficiency is food-cobalamin malabosrption. This typically occurs in older individuals and results from an inability to adequately absorb $\mathrm{Cbl}$ bound in food protein. These patients can absorb free Cbl without difficulty. Therefore, Schilling tests will be normal. No apparent cause of deficiency is identified in a significant number of patients with $\mathrm{Cbl}$ deficiency.

The most common cause of B12 deficiency is pernicious anemia. This autoimmune disorder is characterized by destruction of the gastric mucosa, and the presence of parietal cell and intrinsic factor antibody leading to impaired B12 absorption. The disorder is more common in African-Americans and in patients with Northern European background.

Chronic exposure to nitrous oxide has been associated with subacute combined degeneration [15]. The mechanism by which nitrous oxide induces vitamin B12 deficiency is by 
inactivation of methyl-cobalamin thereby inhibiting the conversion of homocysteine to methionine and methyltetrahydrofolate (MTHF) and 5-methylene-tetrahydrofolate (THF), which are required for myelin sheath protein and DNA synthesis.

\section{Clinical Features}

Vitamin B12 (cobalamin) deficiency is associated with hematologic, neurologic, and psychiatric manifestations. Subacute combined degeneration, neuropsychiatric symptoms, peripheral neuropathy and optic neuropathy are the classic neurological consequences of B12 deficiency. Patients may present with neurological symptoms regardless of a normal hematological picture. The neuropathy associated with B12 deficiency usually begins with sensory symptoms in the feet.

Differentiating vitamin B12 deficiency-related polyneuropathy from cryptogenic sensory polyneuropathy (CSPN) can be difficult on clinical grounds only. Clinical features useful to identify vitamin B12 deficiency related peripheral neuropathy are the acuteness of symptoms onset, and concomitant involvement of upper and lower extremities[16]. Sometimes the sensory symptoms and signs first appear in the upper extremities or the "numb hand syndrome" [17-19]. When this occurs with other findings of a myeloneuropathy, immediately consider B12 as well as copper deficiency (see below). The myeloneuropathy findings often consist of significant proprioception and vibration, increased tone, weakness in a corticospinal tract distribution, (ex. hip and knee flexors), brisk knee and arm reflexes, Hoffman's signs in the fingers, and extensor plantar responses in the toes.

Histopathological studies have showed breakdown and vacuolization of central nervous system myelin under B12 deficiency states [20]. In contrast to the demyelinating features seen in the spinal cord, axonal neuropathy is seen on nerve biopsies and nerve conduction studies in vitamin B12 polyneuropathy.

\section{Diagnosis}

Diagnosis of B12 deficiency is usually made in the presence of typical neurological symptoms, hematological abnormalities, and serum vitamin B12 levels less than $200 \mathrm{pg} / \mathrm{ml}$, though a significant proportion of vitamin B12 deficiency patients may have serum levels that are within the low normal range up to $400 \mathrm{pg} / \mathrm{ml}$. Measurement of the serum metabolites methylmalonic acid (MMA) and homocysteine (Hcy) can improve the sensitivity significantly in patients with low normal range of B12 (300-400 pg/ml) when there is high clinical suspicion [21]. Though elevated MMA and Hcy suggest B12 deficiency, it is necessary to rule out other conditions associated with such abnormal levels, such as renal insufficiency and hypovolemia. Isolated Hcy elevation may also be seen in hypothyroidism, deficiency of folic acid and pyridoxine, cigarette smoking, and advanced age.

Historically, the Schilling test was used to diagnose pernicious anemia. Today, it is difficult to obtain a Schilling test due to the unavailability of the radioisotope. Anti-intrinsic factor and anti-parietal cell antibodies can be helpful in the diagnosis of pernicious anemia with high specificity and low sensitivity for the former and high sensitivity and low specificity 
for the latter. In typical cases with myelopathic symptoms, increased T2 signal intensity is seen in the posterior column on magnetic resonance imaging studies (see imaging below for copper deficiency which is similar).

\section{Treatment [17]}

Early diagnosis is critical since patients with advanced disease may be left with major residual disability. Common treatment regimen includes administration of $1000 \mathrm{mcg}$ intramuscularly daily for 5-7 days, followed by $1000 \mathrm{mcg}$ IM monthly. Other approaches are a once-a-week injections for four weeks, and then monthly injections. Either is probably acceptable. B12 levels should be monitored occasionally to prevent inadequate treatment or non-compliance. Initial severity and duration of symptoms, and the initial hemoglobin measurements correlate with the residual neurological damage after cobalamin therapy. This inverse correlation between severity of anemia and neurologic damage is not understood. If a neurologic response occurs, it does so within the first six months of therapy, although further improvement may occur with time. On the other hand, sometimes treatment only prevents further neurologic impairment, and often patients are left with the neurologic deficits found prior to treatment.

Patients with food-cobalamin malabsorption can absorb free cobalamin and, therefore can be treated with oral cobalamin supplementation. Oral cobalamin replacement therapy may also be an option for patients with pernicious anemia. The daily requirement for cobalamin is 1 to $2 \mu \mathrm{g}$, and approximately $1 \%$ of orally administered cobalamin can be absorbed by patients with pernicious anemia. Therefore, theoretically, an oral cobalamin dose of $1000 \mathrm{mg}$ per day should be sufficient. Although oral cobalamin may seem preferable to intramuscular injections, parenteral therapy is actually less expensive (if it is self-administered). Given the absence of convincing data regarding oral replacement in patients with neurologic deficits, the authors' practice is to use intramuscular cobalamin therapy when the etiology is pernicious anemia. However, a reasonable compromise may be to switch to oral therapy after several months and periodically monitor MMA or Hcy levels.

There is no clear evidence that folic acid therapy precipitates or exacerbates B12 deficiencyrelated neuropathy, however pharmacological doses of folic acid may reverse the hematological abnormalities of cobalamin deficiency, masking early recognition of symptoms, therefore, resulting in the development or progression of neurological symptoms.

\section{Vitamin E Deficiency}

\section{Pathogenesis}

Vitamin E is abundantly available in the diet and is present in animal fat, nuts, vegetable oils and grains. Alpha- tocopherol is the biologically active form of vitamin $\mathrm{E}$ in humans. The RDA of vitamin $\mathrm{E}$ is $15 \mathrm{mg}$ per day of alpha-tocopherol[22]. Dietary vitamin $\mathrm{E}$ is incorporated into chylomicrons and passively absorbed in the intestines. This process requires bile acids, fatty acids, and monoglycerides for absorption[9]. Vitamin E is delivered to tissues via the chylomicrons and then chylomicron remnants when vitamin $\mathrm{E}$ is transferred to very low-density lipoproteins (VLDL) via alpha-tocopherol transfer protein (TTP). Most vitamin E deficiencies occur in patients with malabsorption or transport 
deficiencies. Patients with cystic fibrosis who have malabsorption can develop vitamin E deficiency.

The pathogenesis of vitamin E deficiency is poorly understood. Vitamin E is an antioxidant and a free radical scavenger, and it is postulated that the neurological manifestations of vitamin E deficiency are primarily related to the loss of this protective function. Fat malabsorption is the main cause of vitamin E deficiency. Isolated vitamin E deficiency is a rare autosomal recessive disorder caused by a mutation in the alpha-tocopherol transfer protein gene on chromosome 8q13 [23]. Another hereditary disorder leading to vitamin E deficiency is abetalipoproteinemia, a rare autosomal dominant disorder resulting from mutations in the microsomal triglyceride transfer protein[24]. Patients with this disorder have fat malabsorption and deficiencies of many fat soluble vitamins. If left untreated, patients with this disorder develop pigmented retinopathy, loss of vibration and proproprioception, loss of deep tendon reflexes, ataxia and cerebellar degeneration as well as generalized muscle weakness[25].

\section{Clinical features}

Because alpha- tocopherol is stored in adipose tissues, symptoms of vitamin E deficiency may take 5-10 years to manifest. The onset of symptoms is usually slow and progressive. Clinical features of vitamin E deficiency mimic that of Friederich's ataxia and include ataxia, hyporeflexia, and loss of proprioception and vibration. Other findings on neurological examination may include dysarthria, nystagmus, ophthalmoparesis, retinopathy, head titubation, decreased sensation, and proximal muscle weakness. Pes cavus, and scoliosis may be present. Nerve conduction studies in vitamin E deficiency show a sensory predominant axonal neuropathy. Nerve biopsy shows loss of large myelinated fibers with evidence of regeneration. [26]. Electromyography is often normal, although mild signs of denervation may occur. SSEP may show abnormalities consistent with posterior column involvement [27].The principal pathologic features of vitamin E deficiency include swelling and degeneration of large myelinated axons in the posterior columns, peripheral nerves, and sensory roots[9].

\section{Diagnosis}

Diagnosis is made by measuring alpha-tocopherol levels in the serum. Serum vitamin E levels may be normal even when deficiency is present. The ratio of total serum vitamin $\mathrm{E}$ to the total serum lipid concentration has been suggested as a superior assessment of vitamin $\mathrm{E}$ status[28].

\section{Management}

Treatment of Vitamin E deficiency may reverse or halt the progression of the neurological symptoms. Treatment begins with oral supplementation of Vitamin E 400 international units twice daily, with a gradual increase in the dose until normalization of serum vitamin $\mathrm{E}$ levels. Patients with abetalipoprotienemia may require very large doses of vitamin $\mathrm{E}$ to normalize serum vitamin E levels. Malabsorption syndromes may require treatment with water-miscible or intramuscular preparations of vitamin E. 


\section{Vitamin B6}

\section{Pathogenesis}

Vitamin B6, or pyridoxine, is unique in that either a deficiency or an excess can cause a neuropathy. Pyridoxine is readily available in the diet and dietary deficiency of B6 is rare. Humans are not able to synthesize B6, so dietary intake is essential. After absorption, pyridoxine is converted into pyridoxal phosphate which is an important co-factor in numerous metabolic reactions. The RDA for pyridoxine is $1.3 \mathrm{mg}$ daily with the upper limit of 100mg daily[2]. Doses of 50mg to 100mg of vitamin B6 should mainly be used in certain conditions such as pyridoxine deficient seizures and patients taking certain medications to avoid toxicity.

Vitamin B6 deficiency is most commonly seen in patients treated with the certain medications that are B6 antagonists, namely isoniazid, phenelzine [29], hydralazine [30], and penicillamine. B6 deficiency can also be seen in patients receiving chronic hemodialysis [31]. Vitamin B6 deficiency may also result from the malnutrition due to chronic alcoholism and in patients with high metabolic needs such as the pregnant or lactating woman. Risk factors for vitamin B6 toxicity are excessive intake of supplements $[32,33]$.

\section{Clinical Features}

In infants, pyridoxine deficiency is a cause of seizures. In adults neuropathy due to B6 deficiency starts with numbness, paresthesias, or burning pain in the feet which then ascends to affect the legs and eventually the hands. Neurological examination reveals a length dependent polyneuropathy with decreased distal sensation, reduction of deep tendon reflexes, ataxia and mild distal weakness.

Vitamin B6 toxicity produces a sensory ataxia, areflexia, and impaired cutaneous sensation. Patients often complain of burning or paresthesias. Electrodiagnostic testing usually shows a sensory neuronopathy, but with severe toxicity motor nerves can be affected as well[33]. Symptoms of toxicity can be seen with doses as low as $100 \mathrm{mg}$ per day[34].

\section{Diagnosis}

Vitamin B6 deficiency can be detected by direct assay of blood or urine. Pyridoxal phosphate can also be measured in the blood. Nerve conduction studies reveal severely reduced sensory nerve action potentials with preserved CMAP. Sural nerve biopsy confirms axonal degeneration of small and large myelinated fibers.

\section{Management}

Vitamin B6 supplementation with 50mg per day is suggested for patients being treated with isoniazid or hydralazine. Daily B6 doses of $10 \mathrm{mg}$ to $50 \mathrm{mg}$ are recommended for patients undergoing hemodialysis[31].

The treatment for B6 toxicity is to stop the exogenous B6. Patients may continue to have symptom progression for 2-3 weeks following the discontinuation of vitamin B6 before a gradual improvement starts, a phenomenon known as coasting. 


\section{Pellagra (Niacin Deficiency) \\ Pathogenesis}

Pellagra is the clinical manifestation of nicotinic acid (niacin or B3) deficiency. The classic clinical triad of pellagra is dermatitis, dementia, and diarrhea. Pellagra was once endemic in the United States and Europe and is still occasionally encountered. Most modern patients with pellagra have other risk factors for malnutrition such as homelessness[35], anorexia[36-38], certain cancers, or malabsorption[39].

Niacin is absorbed in the intestine by simple diffusion. The RDA for adults for niacin is $14 \mathrm{mg}$ to $16 \mathrm{mg}$ a day[2]. Niacin and its derivatives are important in carbohydrate metabolism

\section{Clinical Features}

Early neurological symptoms are predominantly neuropsychiatric including apathy, inattention, irritability, and depression. Without treatment symptoms can progress to stupor or coma. Isolated niacin is not known to be a cause of neuropathy and most patients deficient in niacin have other nutritional deficiencies as niacin alone will not improve neuropathy [9].

\section{Diagnosis}

There is no reliable measure of serum niacin.

\section{Management}

Oral replacement of nicotinic acid of $50 \mathrm{mg}$ two or three times a day is recommended for treatment, but dose may be limited due to flushing. Nicotinamide can be used as a substitute in patients unable to tolerate nicotinic acid. Pellagra should be considered in any patient deficient in vitamin B12 or thiamine whose cognition does not improve with supplementation.

\section{Copper Deficiency}

\section{Pathogenesis}

Copper deficiency has long been recognized as a cause of hematologic abnormalities in humans, but neurological abnormalities due to copper deficiency were not reported until 2001 [40]. Since then copper deficiency has been reported to cause either myelopathy or a myeloneuropathy[41]. Copper deficiency has also been reported in association with peripheral neuropathy $[42,43]$, but it is not clear from these case reports if the neuropathy was isolated or in association with other neurological manifestations. Copper sources are common in most western diets and copper rich foods include seafood, nuts, wheat and grains. The RDA for copper for adults is $900 \mathrm{mcg}$ daily[44].

Copper is essential in many oxidative reactions in the body. These reactions can generate free radicals which are toxic to the cell and so both the absorption and excretion of copper are tightly regulated by cells. 
Gastric acid is needed to solubilize dietary copper. Afterward, it is absorbed by both active and passive mechanisms in the intestines. The active transport mechanism predominates when dietary copper is low and augmented by passive diffusion when dietary copper is high. Gastric acid is needed to solubilize dietary copper. Once copper enters the serum it is bound to plasma proteins and transported via the portal vein to the liver. Here copper is incorporated into ceruloplasmin for delivery to cells. If copper supplies are high, the liver excretes excess copper into the bile.

The most common cause of copper deficiency is prior gastric surgery. Exogenous zinc intake from either excessive intakes of zinc supplements [45]or use of older zinc containing denture creams[46, 47]has also been postulated as a cause of copper deficiency with neurological manifestations. Both zinc and copper bind to metallothionein in the enterocytes. Excessive zinc intake leads to up regulation of these complexes and copper has a higher affinity for these receptors than zinc leading copper to displace zinc. The zinc is then absorbed into the bloodstream and the copper/metallothionein complex remains in the enterocyte and is excreted in the feces following normal sloughing of these cells. Copper deficiency can also be seen in association with excess iron consumption and malabsorption syndromes.

\section{Clinical Features}

The majority of patients present with gait difficulty and lower limb paresthesias.

Neurological examination reveals loss of proprioception and vibration due to dorsal column dysfunction and sensory ataxia. Upper motor neuron signs such as bladder dysfunction, brisk knee jerks, and extensor plantar reflexes can also be elicited[41]. A motor neuron disease-like presentation has also been reported [48, 49].

MRI studies of the spinal cord are reportedly abnormal in $47 \%$ of cases showing increased $\mathrm{T} 2$ signal in the posterior columns in both the cervical and thoracic spinal cord[50] [Figure 1]. Neurophysiologic studies are abnormal in most patients with a copper myelopathy. Nerve conduction studies are compatible with mixed, motor and sensory axonal polyneuropathy[41].

\section{Diagnosis}

Hematologic abnormalities are common in patients with copper deficiency, particularly anemia or occasionally myelodysplastic syndrome. In copper deficiency serum copper, ceruloplasmin and urinary excretion of copper will be low, and zinc often will be high. Ceruloplasmin is an acute phase reactant, so this may not be an adequate marker in certain patients.

\section{Management}

In patients with copper deficiency due to excessive zinc intake, it is important to discontinue the exogenous zinc. Replacement with $2 \mathrm{mg}$ of elemental copper three times a day orally is the preferred method of copper replacement. Our clinic combines oral replacement with a 2 mg weekly IV infusion for one month. Copper salts (copper gluconate or copper chloride) may be given intravenously. Hematologic abnormalities due to copper deficiency often 
respond completely and promptly. While copper replacement will stop progression of neurological abnormalities patients are often left with residual symptoms[41].

\section{Neuropathy Following Bariatric Surgery}

Obesity is an increasing medical challenge in both developed and developing counties. In 2010, more than $35 \%$ of Americans were obese, and 5\% of Americans were morbidly obese. Bariatric surgery is an effective procedure for weight loss in morbidly obese patients refractory to a diet and exercise program. More than 200,000 bariatric surgeries were performed in 2008. The number expected to rise with the increase obesity population.

Neurological complications have gained attention in association with bariatric surgery. Neurological complications can involve the entire nervous system ranging from diffuse encephalopathy to peripheral neuropathy to myopathy. Among the neurological complications seen after bariatric surgery, peripheral neuropathies were the most common and may affect up to $16 \%$ of operated patients[51]. There were three dominant peripheral neuropathy patterns seen after bariatric surgery: sensory-predominant polyneuropathy (acute, subacute and chronic), mononeuropathy and radiculoplexopathy, with the first two being more common than the radiculoplexopathy[51]. Onset of symptoms could be subacute to insidious; the time of onset varies from months to years, post-surgery [52,53]. Protracted vomiting and fast weight loss were risk factors to develop peripheral neuropathy after bariatric surgery[51, 54].

Malnutrition was not uncommon for morbidly obese patients prior to their bariatric surgery. Twenty nine percent patients were thiamine deficiency among 379 consecutive patients undergoing bariatric surgery reported by Flancbaum[55]. The most common nutrient deficiencies following bariatric surgery are deficiencies of thiamine, vitamin B12, vitamin E, vitamin D, and copper[56]. Bariatric procedures cause or worsen malnutrition by restriction of intake or combined restriction of intake and impaired absorption. Peripheral neurological complications after bariatric surgery are probably related to multiple nutritional deficiencies. Thiamine deficiency often was seen in painful polyneuropathy post bariatric surgery, which can present without central involvement (encephalopathy). B12 or copper deficiencies were the cause of myeloneuropathy, though data was not consistent [51].

Thiamine, B12 and copper should be a part of baseline metabolic work-up for patient undergoing bariatric surgery, especially patients who were on a diet prior to surgery. Education regarding the importance of adherence to nutritional supplements after surgery is the key to prevent peripheral neuropathy developed post bariatric surgery.

\section{Citations}

1. Butterworth RF. Effects of thiamine deficiency on brain metabolism: implications for the pathogenesis of the Wernicke-Korsakoff syndrome. Alcohol Alcohol. 1989; 24(4):271-9. [PubMed: 2675860]

2. Intakes, A.R.o.t.S.C.o.t.S.E.o.D.R., et al. Dietary Reference Intakes for Thiamin, Riboflavin, Niacin, Vitamin B6, Folate, Vitamin B12, Pantothenic Acid, Biotin, and Choline. The National Academies Press; 1998. 
3. Koike H, et al. Rapidly developing weakness mimicking Guillain-Barre syndrome in beriberi neuropathy: two case reports. Nutrition. 2008; 24(7-8):776-80. [PubMed: 18440777]

4. Murphy C, Bangash IH, Varma A. Dry beriberi mimicking the Guillain-Barre syndrome. Pract Neurol. 2009; 9(4):221-4. [PubMed: 19608771]

5. Blass, J. Basic Neurochemistry. 5. Raven Press; New York: 1994. Vitamin and nutritional deficiencies; p. 749-760.

6. Koike $\mathrm{H}$, et al. Postgastrectomy polyneuropathy with thiamine deficiency is identical to beriberi neuropathy. Nutrition. 2004; 20(11-12):961-6. [PubMed: 15561484]

7. Saperstein, DB.; Barohn, RJ. Polyneuropathy Caused by Nutritional and Vitamin Deficiency. In: Dyck, P., editor. Peripheral Neuropathy. Elsevier; 2005. p. 2051-2062.

8. Floridi A, et al. Thiamine pyrophosphate determination in whole blood and erythrocytes by high performance liquid chromatography. Int J Vitam Nutr Res. 1984; 54(2-3):165-71. [PubMed: 6500839]

9. Kumar N. Nutritional neuropathies. Neurol Clin. 2007; 25(1):209-55. [PubMed: 17324726]

10. Koike $\mathrm{H}$, et al. Postgastrectomy polyneuropathy with thiamine deficiency. J Neurol Neurosurg Psychiatry. 2001; 71(3):357-62. [PubMed: 11511711]

11. Hong CZ. Electrodiagnostic findings of persisting polyneuropathies due to previous nutritional deficiency in former prisoners of war. Electromyogr Clin Neurophysiol. 1986; 26(5-6):351-63. [PubMed: 3023011]

12. Leishear K, et al. Relationship Between Vitamin B12 and Sensory and Motor Peripheral Nerve Function in Older Adults. Journal of the American Geriatrics Society. 60(6):1057-1063. [PubMed: 22690982]

13. Yang YX, Metz DC. Safety of Proton Pump Inhibitor Exposure. Gastroenterology. 139(4):11151127. [PubMed: 20727892]

14. Reinstatler L, et al. Association of biochemical B(1)(2) deficiency with metformin therapy and vitamin B(1)(2) supplements: the National Health and Nutrition Examination Survey, 1999-2006. Diabetes Care. 35(2):327-33. [PubMed: 22179958]

15. Kinsella LJ, Green R. Anesthesia paresthetica: nitrous oxide-induced cobalamin deficiency. Neurology. 1995; 45:1608-1610. [PubMed: 7644061]

16. Saperstein DS, et al. Challenges in the identification of cobalamin-deficiency polyneuropathy. Arch Neurol. 2003; 60(9):1296-301. [PubMed: 12975298]

17. Saperstein DS, Barohn RJ. Peripheral neuropathy due to cobalamin deficiency. Current Treatment Options in Neurology. 2002; 4:197-201. [PubMed: 11931726]

18. Jackson CE, Barohn RJ. Lesion localization of the numb hand syndrome in B12 deficiency. Electroencephalogr Clin Neuro. 1994; 90:46P.

19. Saperstein DS, Jackson CE, Nations SP, Wolfe GI, Barohn RJ. Uncommon neurologic manifestations of pernicious anemia with cobalamin deficiency. Neurology. 2001; 56:A417.

20. Weir DG, Scott JM. The biochemical basis of the neuropathy in cobalamin deficiency. Baillieres Clin Haematol. 1995; 8(3):479-97. [PubMed: 8534958]

21. Saperstein, D.; Barohn, RJ. Neuropathy associated with nutritional and vitamin deficiencies. In: Dyck, T.; PJ; PK, editors. Peripheral Neuropathy. Vol. 2. Elsevier Saunders; 2005. p. 2051-2062.

22. Antioxidants, P.o.D., et al. Dietary Reference Intakes for Vitamin C, Vitamin E, Selenium, and Carotenoids. The National Academies Press; 2000.

23. Jackson CE, Amato AA, Barohn RJ. Isolated vitamin E deficiency. Muscle Nerve. 1996; 19(9): 1161-5. [PubMed: 8761274]

24. Chardon L. Identification of two novel mutations and long-term follow-up in abetalipoproteinemia: a report of four cases. European journal of pediatrics. 2009; 168(8):983-9. [PubMed: 19066957]

25. Muller DPR. Vitamin E and neurological function. Molecular Nutrition \& Food Research. 2010; 54(5):710-718. [PubMed: 20183831]

26. Zouari M, et al. Electrophysiological and nerve biopsy: comparative study in Friedreich's ataxia and Friedreich's ataxia phenotype with vitamin E deficiency. Neuromuscular Disorders. 1998; 8(6):416-425. [PubMed: 9713861] 
27. Puri V, et al. Isolated vitamin E deficiency with demyelinating neuropathy. Muscle Nerve. 2005; 32(2):230-5. [PubMed: 15803482]

28. Sokol RJ, et al. Vitamin E deficiency with normal serum vitamin E concentrations in children with chronic cholestasis. N Engl J Med. 1984; 310(19):1209-12. [PubMed: 6709026]

29. Heller CA, Friedman PA. Pyridoxine deficiency and peripheral neuropathy associated with longterm phenelzine therapy. Am J Med. 1983; 75(5):887-8. [PubMed: 6638055]

30. Raskin NH, Fishman RA. Pyridoxine-deficiency neuropathy due to hydralazine. N Engl J Med. 1965; 273(22):1182-5. [PubMed: 5847557]

31. Corken M, Porter J. Is vitamin B(6) deficiency an under-recognized risk in patients receiving haemodialysis? A systematic review: 2000-2010. Nephrology (Carlton). 2011; 16(7):619-25. [PubMed: 21609363]

32. Silva CD, D'Cruz DP. Pyridoxine toxicity courtesy of your local health food store. Ann Rheum Dis. 2006; 65(12):1666-7. [PubMed: 17105856]

33. Gdynia HJ, et al. Severe sensorimotor neuropathy after intake of highest dosages of vitamin B6. Neuromuscul Disord. 2008; 18(2):156-8. [PubMed: 18060778]

34. So, YTS.; Roger, P. Deficiency Diseases of the Nervous System. In: Bradley, WD.; Robert; Fenichel, Gerald; Jankovic, Joseph, editors. Neurology in Clinical Practice. ButterworthHeinemann; 2008. p. 1643-1656.

35. Kertesz SG. Pellagra in 2 homeless men. Mayo Clin Proc. 2001; 76(3):315-8. [PubMed: 11243279]

36. Jagielska G, Tomaszewicz-Libudzic EC, Brzozowska A. Pellagra: a rare complication of anorexia nervosa. Eur Child Adolesc Psychiatry. 2007; 16(7):417-20. [PubMed: 17712518]

37. Prousky JE. Pellagra may be a rare secondary complication of anorexia nervosa: a systematic review of the literature. Altern Med Rev. 2003; 8(2):180-5. [PubMed: 12777163]

38. Rapaport MJ. Pellagra in a patient with anorexia nervosa. Arch Dermatol. 1985; 121(2):255-7. [PubMed: 3977345]

39. Jarrett P, et al. Pellagra, azathioprine and inflammatory bowel disease. Clin Exp Dermatol. 1997; 22(1):44-5. [PubMed: 9330055]

40. Schleper B, Stuerenburg HJ. Copper deficiency-associated myelopathy in a 46-year-old woman. J Neurol. 2001; 248(8):705-6. [PubMed: 11569901]

41. Kumar N. Copper deficiency myelopathy (human swayback). Mayo Clin Proc. 2006; 81(10):137184. [PubMed: 17036563]

42. Gregg XT, Reddy V, Prchal JT. Copper deficiency masquerading as myelodysplastic syndrome. Blood. 2002; 100(4):1493-5. [PubMed: 12149237]

43. Willis MS, et al. Zinc-induced copper deficiency: a report of three cases initially recognized on bone marrow examination. Am J Clin Pathol. 2005; 123(1):125-31. [PubMed: 15762288]

44. Micronutrients, P.o., et al. Dietary Reference Intakes for Vitamin A, Vitamin K, Arsenic, Boron, Chromium, Copper, Iodine, Iron, Manganese, Molybdenum, Nickel, Silicon, Vanadium, and Zinc. The National Academies Press; 2001.

45. Rowin J, Lewis SL. Copper deficiency myeloneuropathy and pancytopenia secondary to overuse of zinc supplementation. J Neurol Neurosurg Psychiatry. 2005; 76(5):750-1. [PubMed: 15834043]

46. Nations SP, et al. Denture cream: an unusual source of excess zinc, leading to hypocupremia and neurologic disease. Neurology. 2008; 71(9):639-43. [PubMed: 18525032]

47. Hedera $\mathrm{P}$, et al. Myelopolyneuropathy and pancytopenia due to copper deficiency and high zinc levels of unknown origin II. The denture cream is a primary source of excessive zinc. Neurotoxicology. 2009; 30(6):996-9. [PubMed: 19732792]

48. Weihl CC, Lopate G. Motor neuron disease associated with copper deficiency. Muscle Nerve. 2006; 34(6):789-93. [PubMed: 16929546]

49. Dimachkie, M.; Estephan, B.; Barohn, RJ., et al. Copper Deficiency Myeloneuropathy-Case presentation at the Unusual Diagnostic and Management of Cases in Neuromuscular Disease. American Academy Annual Meeting; April 2011; Honolulu, HI.

50. Jaiser SR, Winston GP. Copper deficiency myelopathy. J Neurol. 2010; 257(6):869-81. [PubMed: 20232210] 
51. Thaisetthawatkul $\mathrm{P}$, et al. A controlled study of peripheral neuropathy after bariatric surgery. Neurology. 2004; 63(8):1462-70. [PubMed: 15505166]

52. Abarbanel JM, et al. Neurologic complications after gastric restriction surgery for morbid obesity. Neurology. 1987; 37(2):196-200. [PubMed: 3027610]

53. Juhasz-Pocsine K, et al. Neurologic complications of gastric bypass surgery for morbid obesity. Neurology. 2007; 68(21):1843-50. [PubMed: 17515548]

54. Halverson JD. Metabolic risk of obesity surgery and long-term follow-up. Am J Clin Nutr. 1992; 55(2 Suppl):602S-605S. [PubMed: 1733137]

55. Flancbaum L, et al. Preoperative Nutritional Status of Patients Undergoing Roux-en-Y Gastric Bypass for Morbid Obesity. Journal of Gastrointestinal Surgery. 2006; 10(7):1033-1037. [PubMed: 16843874]

56. Becker DA, Balcer LJ, Galetta SL. The Neurological Complications of Nutritional Deficiency following Bariatric Surgery. J Obes. 2012; 2012:608534. [PubMed: 22970351] 


\section{Key Points}

- Neuropathies due to nutritional problems can affect certain patient populations and have a varied presentation due to multiple co-existent nutritional deficiencies.

- Clinicians should consider nutritional neuropathies in patients presenting with neuropathies.

- Clinicians should be alert for signs and symptoms of neuropathy in patients who have had bariatric surgery. 

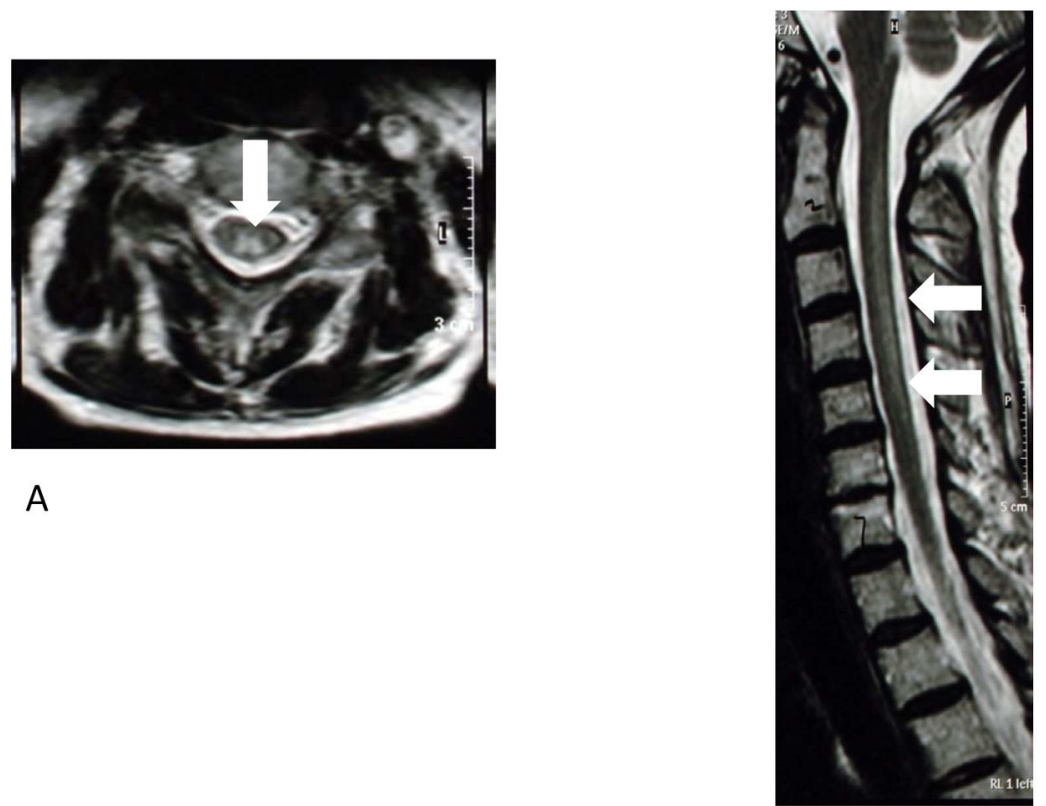

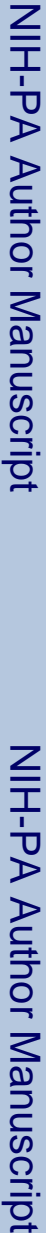

A

B

Figure 1.

Axial (A) and sagittal (B) T2 weighted MRI scans demonstrating posterior column hyperintensity (arrows). 